\title{
EXPERIENCIAS, VALORES Y PREFERENCIAS DE LAS PERSONAS CON TRASTORNO DE ANSIEDAD GENERALIZADA SOBRE EL CUIDADO DE SU SALUD: INVESTIGACIÓN CUALITATIVA
}

\author{
Vanesa Ramos-García1, Ana Toledo-Chávarri', María del Mar Trujillo-Martín', Tasmania Del \\ Pino-Sedeño ${ }^{1}$, Wenceslao Peñate Castro ${ }^{2}$ y Lilisbeth Perestelo Pérez ${ }^{3}$ \\ ${ }^{1}$ Fundación Canaria Instituto de Investigación Sanitara de Canarias (FIISC), España. vanesa.ramosgarcia@sescs.es; \\ anatoledochavarri@sescs.es; mar.trujillomartin@sescs.es; tasmania.delpino@sescs.es \\ ${ }^{2}$ Facultad de Ciencias de la Salud - Sección de Psicología, Universidad de La Laguna (ULL), España. wpenate@ull.edu.es \\ ${ }^{3}$ Servicio de Evaluación del Servicio Canario de La Salud (SESCS), España. lilisbeth.peresteloperez@sescs.es
}

\begin{abstract}
Resumen. El Trastorno de Ansiedad Generalizada (TAG) es uno de los trastornos de ansiedad más comunes y representa un porcentaje elevado en el ámbito de atención primaria (AP). Conocer los valores y preferencias de las personas con TAG en relación a los tratamientos disponibles facilita su elección y mejora las condiciones de salud. Objetivo: Realizar un estudio cualitativo para conocer la experiencia, valores y preferencias de las personas con TAG respecto a los tratamientos e incluir los resultados en las recomendaciones de una Guía de Práctica Clínica. Método: Diseño cualitativo con entrevistas semiestructuradas. Resultados: A partir del análisis narrativo se identificaron 5 temas: 1) Acceso al cuidado: diagnóstico, tratamiento farmacológico y atención especializada; 2) Aceptabilidad de los tratamientos; 3) Valores y preferencias; 4) La atención en atención primaria; 5) Conocimien0to para la autogestión y necesidades de información. Conclusiones: La aceptabilidad de los tratamientos es variable y el diagnóstico es más frecuente fuera de entornos de AP. Existe una necesidad de información en relación al TAG y a sus tratamientos.
\end{abstract}

Palabras-clave: Atención Primaria; Autocuidado; Trastorno de Ansiedad Generalizada; Valores y Preferencias.

\section{EXPERIENCES, VALUES AND PREFERENCES OF PEOPLE WITH GENERALIZED ANXIETY DISORDER ABOUT THEIR HEALTH CARE: QUALITATIVE RESEARCH}

\begin{abstract}
Generalized Anxiety Disorder (GAD) is one of the most common anxiety disorders and represents a high percentage in primary care (PC) settings. Knowing the values and preferences of people with GAD in relation to available treatments facilitates their choice and improves health conditions. Goals: To conduct a qualitative study to learn about the experience, values and preferences of people with GAD with respect to treatments and to include the results in the recommendations of a CPG. Method: Design qualitative with semi-structured interviews. Results: Five topics were identified from the narrative analysis: 1) Access to care: diagnosis, drug treatment and specialized care; 2) Acceptability of treatments; 3) Values and preferences; 4) Care in primary care; 5) Knowledge for self-management and information needs. Conclusions: Acceptability of treatments is variable and diagnosis is more frequent outside PC settings. There is a need for information regarding GAD and its treatments.
\end{abstract}

Keywords: Primary Care; Self-Care; Generalized Anxiety Disorder; Values and Preferences. 


\section{INTRODUCCIÓN}

Los trastornos de ansiedad (TA) son el tipo de trastorno mental más frecuente, con una prevalencia doble en las mujeres que en los hombres (World Health Organization 2017; D. J. Stein et al. 2017). Afectan a más de 260 millones de personas (Organización Mundial de la Salud, 2017) y aproximadamente a 61.5 millones de adultos en Europa (Begum et al. 2020), mientras que en España, un estudio reciente indica que afectan al 11\% de personas (Villagrasa et al. 2019). Los TA son los más frecuentes dentro del ámbito de Atención Primaria (AP) y concretamente, el trastorno de ansiedad generalizada (TAG) tiene una alta prevalencia (Alonso and Lépine 2007; Bados 2017).

La característica esencial del TAG es una excesiva, persistente y exagerada preocupación por los acontecimientos de la vida cotidiana, sin razón aparente. Durante al menos 6 meses la persona experimenta episodios de angustia con preocupación excesiva, caracterizados por una sensación de "intranquilidad", fatiga crónica, irritabilidad o tensión muscular y presenta dificultades para manejar la incertidumbre, ya que a menudo se condicionan por el peligro o amenazas externas sin existir razón aparente (American Psychiatric Association 2013).

Con frecuencia, el TAG se presenta junto a otros trastornos del estado de ánimo y TA (den Boeft et al. 2016; Meuldijk et al. 2016), y especialmente, tiene una alta correlación con los trastornos depresivos (Parmentier, García-Campayo, and Prieto 2013). Debido a su alta comorbilidad, las personas con TAG suelen acudir a los servicios de AP por síntomas somáticos físicos o relacionados con su ansiedad, tales como dolores de cabeza o de espalda, problemas gastrointestinales (dispepsia), cardiovasculares (palpitaciones) 0 respiratorios (disnea) (García-Campayo, Caballero, Perez, \& López, 2012; Stein \& Sareen, 2015); lo cual dificulta su diagnóstico desde AP en fases tempranas del trastorno y, por consiguiente, la elección de un tratamiento adecuado para mejorar las condiciones de salud.

Las herramientas de ayuda a la toma de decisión (HATD) son intervenciones que apoyan a los pacientes a hacer explícitas sus decisiones, proporcionan información acerca de las opciones y beneficios de cada opción de tratamiento y ayudan a aclarar los valores personales (Stacey et al. 2017). De este modo, cuando el paciente es partícipe del proceso de decisión del tratamiento junto al profesional sanitario, y se tienen en cuenta las experiencias, valores y preferencias del paciente, aumenta la satisfacción en el proceso de 
toma de decisión conjunta del tratamiento, así como el conocimiento sobre su enfermedad (Stacey et al. 2017; Légaré et al. 2018).

En este sentido, conocer de antemano las experiencias, valores y preferencias de las personas con TAG, facilita el proceso de tomar una decisión conjunta con el profesional sanitario respecto a las opciones de tratamientos disponibles. Además de las HATD, las Guías de Práctica Clínica (GPC) incorporan los valores y preferencias de la población, teniendo presente factores como la equidad, la aceptabilidad y viabilidad para determinar la fuerza de las recomendaciones (van der Weijden et al. 2012) y adaptando las recomendaciones basadas en la población en recomendaciones dirigidas a personas de manera individual (van der Weijden et al. 2012).

El objetivo de este estudio es incorporar en la actualización de una GPC para el tratamiento del TAG en AP (en proceso de elaboración), coordinada por el Servicio de Evaluación y Planificación del Servicio Canario de la Salud (SESCS) y Guía Salud (RedETS) las experiencias, valores y preferencias de las personas con TAG, con el fin de ayudar a los profesionales sanitarios de AP, a las personas con TAG y sus familias a tomar decisiones más informadas sobre cómo abordar su problema de salud y los tratamientos disponibles.

\subsection{Objetivo principal}

Realizar un estudio cualitativo que permita conocer las experiencias, valores y preferencias de las personas con TAG e incluir los resultados en las recomendaciones de la GPC.

\subsection{Objetivos secundarios}

1) Conocer cuál es la atención que reciben las personas con TAG en el ámbito de atención primaria; 2) Explorar los valores y preferencias de las eprsonas con TAG con respecto a los diferentes tratamientos y 3 ) identificar cuáles son las necesidades de información de las personas con TAG.

\section{MÉTODO}

\subsection{Diseño del estudio}

Se siguió una metodología cualitativa a partir de entrevistas semiestructuradas. 


\subsection{Contexto}

Las entrevistas semiestructuradas se llevaron a cabo de forma presencial o telefónica en el centro de salud de referencia de los participantes.

\subsection{Criterios de inclusión y exclusión}

Inclusión: Personas adultas, mayores de 18 años y con un diagnóstico principal de TAG realizado por los servicios de AP (Sistema Nacional de Salud) o en una consulta privada. Exclusión: Personas con otro TA (agorafobia con o sin trastorno de pánico) como diagnóstico principal, personas con TAG con alguna enfermedad crónica incapacitante o con un trastorno cognitivo que dificultase la participación en la entrevista.

\subsection{Muestreo}

Los participantes se seleccionaron mediante un muestreo intencional de máxima variación, con el objetivo de cubrir las variaciones individuales más relevantes en relación a las experiencias, valores y preferencias con los distintos tratamientos. Se incluyeron personas que hablaran español, mayores de edad y menores 65 años, de ambos géneros, con un tiempo desde el diagnóstico reciente ( $<5$ años) y no reciente ( $>5$ años) y tratados, mayormente, en AP.

\subsection{Reclutamiento}

El equipo investigador elaboró un formulario online -Google Forms- donde se explicitó el objetivo de dicho estudio y se pidió la colaboración voluntaria de la población de las distintas Comunidades Autónomas (CC.AA). Dicho enlace se distribuyó a través de las redes sociales (Twitter y Facebook) de la Red Española de Agencias de Evaluación de Tecnologías Sanitarias (RedETS), prestaciones del Servicio Nacional de Salud (SNS) y de la asociación de pacientes AMADAG (Asociación Madrileña de Agorafobia). Aquellas personas que cumplieron criterios se les citó para la entrevista.

\subsection{Instrumento de recogida de datos}

El guion semiestructurado cubrió los siguientes aspectos: 1) Trayectoria general de la enfermedad, experiencia pre-diagnóstica, diagnóstica, sintomatología y comorbilidades; 2) Experiencia con los tratamientos, opciones de tratamientos recibida, acceso y adherencia a 
esos tratamientos; 3) Aceptabilidad y preferencias con respecto a los tratamientos y la toma de decisiones compartida; 4) Importancia de las medidas de resultado (valoración individual); 5) Medidas de resultado de los tratamiento (grado de importancia individual atribuida a los tratamientos en relación a la frecuencia, duración e intensidad de los síntomas); 6) AP (exploración de la atención sanitaria recibida en atención primaria, diagnóstico, recaídas y tratamientos); 7) Necesidades de información (exploración sobre el conocimiento y aprendizaje del trastorno, información recibida en AP o búsqueda de información fuera de AP); 8) Apoyo emocional y social del entorno de la persona; y 9) Recomendación a otras personas que estén recién diagnosticadas de TAG.

\subsection{Análisis de los datos}

Las entrevistas fueron grabadas en audio y transcritas para su análisis. Una investigadora llevó a cabo el proceso de análisis de contenido temático, codificando las transcripciones sistemáticamente a partir del guion previo desarrollado. De esta forma se identificaron los temas, estableciendo conexiones temáticas entre ellos y relacionándolos con las preguntas y los objetivos del estudio (Ritchie, J., Lewis, J., Nicholls, C.M. Ormston 2014). Los temas principales surgidos se discutieron con el equipo investigador. El análisis de las entrevistas se realizó con un software específico para datos cualitativos (NVIVO 12).

\subsection{Análisis éticos}

Este estudio fue desarrollado dentro del marco de actualización de la GPC para el tratamiento del TAG en AP, coordinado por el SESCS y Guía Salud (Grupo de Trabajo de la Guía de Práctica Clínica para el Manejo de Pacientes con Trastornos de Ansiedad en Atención Primaria 2008) siguiendo un enfoque de empoderamiento de pacientes y de atención centrada en la persona. Ambos enfoques éticos (Entwistle and Watt 2013) implican "respetar y responder a las preferencias, necesidades y valores individuales de los pacientes, y garantizar que los valores de los pacientes guíen todas las decisiones clínicas" (Institute of Medicine (IOM) 2001).

La participación fue voluntaria y se pudo abandonar el estudio en cualquier momento. A los participantes se les ofreció tiempo para leer la hoja informativa del estudio y se aseguró que comprendieran su participación. Antes de cerrar una cita, los participantes tuvieron la oportunidad de hacer preguntas antes de decidir si participar o no y firmar su consentimiento. 
Las medidas para el tratamiento, la comunicación y la cesión de los datos de carácter personal de todos los participantes se ajustó a lo dispuesto en la Ley Orgánica 3/2018, de 5 de diciembre de Protección de Datos Personales y garantía de los derechos digitales, y a la aplicación de del Reglamento (UE) 2016/679 del Parlamento europeo y del Consejo de 27 de abril de 2016 de Protección de Datos (RGPD), los nombres se cambiaron por seudónimos tras el proceso de transcripción para garantizar el anonimato; los datos fueron almacenados de forma segura para garantizar la confidencialidad.

\section{RESULTADOS}

Participaron un total de 8 personas con diagnóstico activo de TAG de las CC. AA de Canarias, Castilla y León, Extremadura y Madrid con edades entre 30 y 65 años; 5 mujeres y 3 hombres. El tiempo desde el inicio del diagnóstico varió entre 1 y 20 años. Siete de los ocho habían sido tratados en atención primaria. Siete personas estaban en tratamiento farmacológico y psicológico, mientras que sólo uno estaba en tratamiento farmacológico. Cuatro personas tenían un diagnóstico comórbido de depresión, otros dos con TA (agorafobia y agorafobia con ataque de pánico), una con un trastorno de la conducta alimentaria y la última no tenía comorbilidades.

El análisis narrativo del estudio primario permitió identificar 5 temas principales: 1) Acceso al cuidado: diagnóstico, tratamiento farmacológico y atención especializada; 2) Aceptabilidad de los tratamientos; 3) Valores y preferencias; 4) La atención en AP; 5) Conocimiento para la autogestión y necesidades de información. A continuación, se destacan los principales resultados por áreas temáticas.

La trayectoria diagnóstica del TAG es variable. Generalmente, los profesionales de psiquiatría y psicología son quienes diagnostican TAG, aunque la mayoría de las veces, la ansiedad se identifica en los servicios de urgencias o en AP, donde las crisis agudas que sufren las personas con ansiedad suelen ser clave para su diagnóstico.

Sin embargo, las personas con TAG acuden a estos servicios buscando un origen no psicológico a sus síntomas. En este sentido, el diagnóstico de TAG en AP juega un papel limitado, ya que ninguna de las personas entrevistadas recibió el diagnóstico de TAG en AP. 
Cuadro 1. Tema 1. Acceso al cuidado: diagnóstico, tratamiento farmacológico y atención especializada.

\section{Citas}

"El médico de cabecera se lo comenté y me derivó directamente a psiquiatría, él no me dijo nada". TAG01MADLG35M

“(...)Acudí a urgencias por ese tema, me hacian pruebas (...)Y eso fue así como durante... pues tranquilamente uno o dos meses, uno o dos meses hasta que una vez estuve en urgencias y me acuerdo que fue una doctora que me dijo... sacó un blister de pastillas y me dijo: "Mira, te vas a meter esta pastilla abajo de la lengua y vas a esperar fuera en la sala de espera durante 30 minutos, atiendo otro paciente y te vuelvo a llamar". "(...) Entonces automáticamente me derivó al psiquiatra y al psicólogo para que me valoraran un poquito más en profundidad, y ya me... fue cuando me pusieron a tratamiento para la ansiedad. TAG04CYLRSR38H

El proceso diagnóstico puede llevar desde pocos días o meses -en crisis aguda- o alargarse durante años (4 de las 8 personas entrevistadas, obtuvieron el diagnóstico después de 10 años, después del debut de los problemas y, de éstas, una más después de 20 años y otra después de 30 años).

Cuadro 2. Tema 1. Acceso al cuidado: diagnóstico, tratamiento farmacológico y atención especializada.

\section{Citas}

"Pues tardé muchísimos años en saber qué es lo que me pasaba”. "(...) Yo empecé con 16 años, tengo 44, y lo que yo recuerdo es que prácticamente me vino de la noche al día (...)". TAG02ME44M

"Pues yo comencé con la ansiedad cuando tenía como 9 años. No se sabía realmente en aquella época, ya que tengo 52 años para 53, no se sabía realmente por qué actuaba de esa manera pero un día era muy nerviosa, siempre lloraba por todo, tenía mucha irritabilidad, mucha autoexigencia y luego sobre todo en esa etapa de los 9 años se me presentó una situación con la alimentación..." TAG03CANZHQ39M

El acceso a la atención sanitaria de personas con TAG no es equitativo. La derivación a servicios de psiquiatría, psicología y otros (trabajo social) es variable. Los períodos de espera para la derivación a psicología o psiquiatría son largos y las citas no tienen una periodicidad regular que requeriría el tratamiento de una persona con TAG. 
El acceso a los fármacos es más fácil, aunque también parece haber poca equidad en la capacidad de los médicos de AP para recetar el tratamiento adecuado a las personas con TAG. Tres de los entrevistados perciben que los tratamientos recibidos en AP no les resultan efectivos, y, en un caso, los considera erróneos.

Cuadro 3. Tema 1. Acceso al cuidado: diagnóstico, tratamiento farmacológico y atención especializada.

\section{Citas}

"yo voy a un centro especializado (...) Centro de Rehabilitación Psicosocial. Es gratuito, (...), a mí me ve cada 15 días una psicóloga, no me ha visto durante semanas muchas veces hasta que yo he llegado a estar bien" (...) TAG05BADMAT50M

“(...) otra vez volví a mi médica de cabecera, me volvió a derivar al psicológico y al psiquiatra. TAG04CYLRSR38H

"Mi médico pues una vez me manda el fármaco, él sin ningún problema pues me hace la receta y bueno, ya está de acuerdo en que esa sea la medicación. (...) No he tenido problema en ese aspecto, ni tampoco me ha dicho: "No, mejor esto no", porque obviamente si sabe que me están tratando por otro lado él se ajusta a lo que me digan". TAG03CANZHQ39M.

A las personas con TAG les cuesta aceptar los nuevos tratamientos y cuidados en un primer momento, mostrando resistencia ante ello. Para la mayoría, la resistencia inicial se supera tras un periodo inicial, aunque en otras, esta resistencia es mayor, prologándose en el tiempo y dificultando el acceso al cuidado a largo plazo.

Una persona con TAG pasa por distintas fases diagnósticas sobre las que deben tomar decisiones terapéuticas, y, esperan que la atención sanitaria que reciben de su sistema de salud, tenga en cuenta la temporalidad del trastorno. Sin embargo, el seguimiento a largo plazo de la medicación o de la psicoterapia no parece ser sistemático en el SNS, por lo que no siempre es posible que haya una adaptación a las distintas fases diagnósticas de la enfermedad.

La resistencia a los tratamientos es más común en el caso de los tratamientos farmacológicos, aunque es variable. Algunas personas aceptan permanentemente el fármaco después del rechazo inicial, aunque tengan efectos secundarios que consideren difíciles de llevar (i.e. subir de peso). 
La resistencia a tomarlos puede permanecer latente y se reactiva en momentos en las fases de la enfermedad, pudiendo afectar a las intenciones de interrumpir el tratamiento. Por otro lado, la interrupción del tratamiento farmacológico lo realizan con o sin apoyo médico.

Con apoyo médico, la interrupción es progresiva, mientras que sin apoyo es brusca, pudiendo implicar síntomas causados por la dependencia a los fármacos (vómitos, sudores). Algunas personas justifican la interrupción del tratamiento bien por la sensación de encontrarse bien, o bien por la sensación de sentir dependencia a los fármacos. Las recaídas son muy comunes cuando interrumpen o no el tratamiento farmacológico.

Cuadro 4. Tema 2. Aceptabilidad de los tratamientos.

\section{Citas}

"Y la medicación pues no me la tomé (...) como no me lo tomé tan en serio como que era una enfermedad, pues mejoré bastante sin tratamiento. El tratamiento yo lo comienzo cuando no trago (cuando os síntomas aumentaron)". TAG03CANZHQ39M "con los fármacos tuve un poco de resistencia al principio, sobre todo, con el primer tratamiento (...) pues claro, tienes 21 años y no conoces a nadie que padezca esto y tú no sabes muy bien tampoco lo que es ansiedad, entonces tú piensas que estás loco."

\section{TAG04CYLRSR38H}

"Voy al médico, y "¿Cómo te encuentras?" Bien. "Pues nada, sigue con ello". (...) muchas veces me ha preguntado el médico que si no he pensado en dejar de tomármela, pero la verdad es que no me lo he planteado el dejar de tomármela". TAG01MADLG35M

“(...) estoy con un psiquiatra que él ahora mismo está empezando a retirar poco a poco algunos de los medicamentos (...) sobre todo la adicción que producen (...) los ansiolíticos que he estado tomando. (...) cuesta mucho dejarlos". TAG07CANICR37M “¿Has pensado en algún momento dejar la medicación? R: Jamás (...) a mí el hecho de tomar medicación me ha producido un aumento de peso (...) Pues aun así yo he considerado que tenía que seguir tomando la medicación si quería estar bien (...)".

\section{TAG05BADMAT50M}

“¿Has pensado en algún momento dejar la medicación? R: Sí, sí. Muchas veces. Muchísimas. Muchísimas, porque en el momento que yo veía que avanzaba y luego me iba para atrás, yo decía: ¿Para qué me sirve esto que me estoy tomando?”. TAG06MADAGD 
“Como me encontré bien pues la dejé yo. Claro, y a los seis meses volví a caer, volví otra vez con los miedos, con las fobias, mis amigas decían de ir por ejemplo a un concierto y yo no quería ir (...)". TAG01MADLG35M

La psicoterapia tiene mayor aceptabilidad que los fármacos. Entre las características que la hacen más aceptable, se encuentran: la accesibilidad de los profesionales en momentos de crisis (aumentar el número de sesiones o acceso telefónico); si reciben sesiones terapéuticas semanales durante la primera fase del trastorno.

Según los entrevistados, la aceptabilidad de técnicas como la relajación, meditación, mindfulness, respiración, yoga o autoayuda, está limitada por la necesidad de pasar la barrera de resistencia inicial ante un nuevo tratamiento.

Las personas que practican la relajación, meditación o mindfulness, señalan que han tenido que superar esas barreras iniciales para aceptarlas y aprender a gestionar los síntomas del TAG.

Cuadro 5. Tema 2. Aceptabilidad de los tratamientos.

\section{Citas}

"Yo creo que la psicoterapia es más efectiva en los casos en que no tengamos otro tipo de trastornos, (..). Pero yo creo que debe prevalecer la psicoterapia. (...) Porque la psicoterapia te da todas las herramientas que necesitas (...)". TAG03CANZHQ39M

"Claro, yo al principio también era muy negativista con el tema este de la meditación, pero sí que me ayuda bastante". TAG04CYLRSR38H

"yo desde hace bastantes años he estado, aunque no he progresado en ello, en el tema de las técnicas de relajación, no he progresado mucho” (...). TAG08CANJUV65H

Los entrevistados señalan una clara preferencia por los tratamientos combinados (fármacos y psicoterapia). Sin embargo, algunas prefieren la psicoterapia frente a los fármacos, aunque no renunciarían a éstos si son necesarios.

Por el contrario, ninguna persona entrevistada mostró preferencia por los fármacos exclusivamente. Existe poca información sobre tratamientos farmacológicos concretos. Dentro de los mencionados, la Paroxetina es el fármaco más aceptado y el más eficaz de los probados, ya que suele tomarse tras otros intentos con otro fármaco. 
Cuadro 6. Tema 3. Valores y preferencias.

\section{Citas}

Creo que la psicoterapia la valoro, porque empiezas a conocer por qué le pasa lo que le pasa a tu cuerpo...No es que yo esté en contra de que te tengan que mandar los fármacos, pero es donde empiezas a conocer por qué le pasa cada cosa, de dónde puedes venirte esos problemas, e incluso a controlarlos a través de respiraciones, de ejercicios y demás (...)". TAG07CANICR37M

"Preferiría la psicoterapia. (...) yo soy una persona súper reacia, que me da miedo tomarme antidepresivos". TAG06MADAGD

"No. Son tratamientos combinados, estoy a favor del tratamiento combinado, medicación y terapia. Nunca por separado (...). Tú tienes que tomar tu medicación, pero la medicación sola no hace nada". TAG05BADMAT50M

En general, la valoración de la AP en el cuidado del TAG no es positiva. Sólo una de las personas entrevistadas tiene a su médico de AP como referencia en el cuidado de su enfermedad y acude a su profesional de medicina para gestionar sus crisis, recibir tratamiento farmacológico y derivación a psiquiatría.

La mayoría de las personas con TAG tienen como referente de cuidado los profesionales de psiquiatría o psicología, que, en muchas ocasiones, no trabajan en el SNS.

Las personas entrevistadas valoran como negativo la falta de tiempo durante las consultas, la falta de conocimiento y empatía sobre el TAG que pueden tener algunos profesionales del sistema sanitario, así como una ausencia de la respuesta del sistema sanitario ante los momentos de crisis, ante la falta de pautas y planes de actuación para dichas fases agudas.

La falta de conexión entre AP y los servicios especialistas de psiquiatría y psicología es otro aspecto que consideran negativo.

Los entrevistados valoran positivamente las situaciones en las que los profesionales de medicina de AP realizan un acompañamiento durante las fases de la enfermedad, que puede incluir el seguimiento a largo plazo del tratamiento con fármacos, así como brindar información acerca de los fármacos, sus efectos y la posibilidad de interrumpir el fármaco tras procesos continuos de recaídas. 
Cuadro 7. Tema 4. La atención en atención primaria.

\section{Citas}

"Yo fui por una cuestión porque bueno, fui primero al médico de la Seguridad Social, el cual no me fue bien": TAG01MADLG35M

“¿Por tanto considera que hay carencias en la atención? R: A mí desde luego sí. Y al día de hoy igual. Primero, lo muchísimo que se tarda en atender a una persona, he tenido momentos realmente cruciales en mi vida $y$ no he sido atendida como consideraba, ni saben consolar a una persona ni saben ser empáticas”. TAG02ME44M "mi médico pues debe llevar conmigo como ya siete u ocho años. Pues ya me conoce bastante (...). Sí que he notado como una atención más cercana, y como que conocen mucho mejor mi caso, que pueden conocer... tanto el psiquiatra como el psicólogo, como la psicóloga que yo tengo". TAG04CYLRSR38H

"Por lo menos la mía, todos los médicos de Atención Primaria al final hacen un poquito de psicólogos. (...) Entonces yo creo que sí, que desde Atención Primaria se puede hacer mucho más. Lo que pasa que los médicos de cabecera al final con pasar sus consultas y todo eso, yo creo que ya tienen el tiempo cubierto". TAG04CYLRSR38H

Las entrevistas realizadas indican importantes necesidades de información sobre el TAG, sus síntomas, los tratamientos disponibles, riesgos y beneficios asociados al ellos.

También se han encontrado beneficios para la autogestión del conocimiento sobre el TAG. En este sentido, conocer la definición del TAG, los síntomas específicos que tiene cada persona, tomando consciencia de que pueden cambiar con el tiempo o reaparecer cuando hay un curso largo de la enfermedad, puede ayudar a la autogestión de los propios síntomas.

Los síntomas pueden disminuir con el conocimiento de la propia enfermedad y emplear herramientas y estrategias psicológicas o de autogestión en función de cada persona. Se detectó la necesidad de conocer los efectos secundarios y la posibilidad de dependencia de alguno de los tratamientos farmacológicos.

Se desconoce que algunos fármacos requieren seguimiento a largo plazo y una planificación sobre cuándo y cómo debe interrumpirse el tratamiento. 
Cuadro 8. Tema 5. Conocimiento para la autogestión y necesidades de información.

\section{Citas}

"Al inicio no eres tan consciente, pero cuando se te va pasando un poco, (...), ves una mejoría, empiezas a investigar por tu cuenta, qué es la agorafobia, por ejemplo, qué es el TAG, qué síntomas (...), el hecho de saber que los síntomas, en este caso sobre todo con la ansiedad generalizada y con determinados pensamientos obsesivos, (...) más te crecen esos pensamientos y más te crece la ansiedad (...). Para mí, esa información fue fundamental (...)”. TAG06MADAGD

"En atención primaria, la verdad que no me lo explicaron, me lo explicó la psicóloga. Me explicó en qué consistía, un poco los síntomas... (...)". TAG03CANZHQ39M

"Nadie te explicó nada. No, no, pues es ansiedad pero nadie te dice... Pues mira, vas a tener este síntoma y esto y esto, las explicaciones son muy...(...)". TAG04CYLRSR38H "Me hubiera encantado que en el primer momento me hubieran dicho "Mira, tienes un trastorno de ansiedad generalizada y una depresión severa mayor y el trabajo que vamos a tener que seguir es este", pero no lo hicieron". TAG05BADMAT50M

“(...) Yo voy donde mi médica y le digo: Mira, E., esta medicación que mandaste me provoca a lo mejor estos síntomas que no sé si son normales". TAG04CYLRSR38H

"Poco a poco, [los síntomas] han ido como disminuyendo en medida, ya, gracias al conocimiento, todo lo que me han explicado, lo que no es que no me asustan los síntomas". TAG06MADAGD

"Pero son truquitos que vas descubriendo tú, pues eso, de vivir 20 años con... (...) Y luego esos trucos por ahí veces que dejan de funcionar, no sabes por qué". TAG04CYLRSR38H

\section{CONCLUSION}

Este estudio primario cualitativo ha analizado la experiencia, valores y preferencias de las personas con TAG en relación con los tratamientos recibidos. Los resultados han mostrado que el proceso de diagnóstico del TAG es más frecuente en los servicios de urgencias o en atención especializada, aunque el acceso a los servicios sanitarios parece no ser equitativo para todas las personas. Este hallazgo es congruente con otros estudios, donde existe una ligera tendencia a acudir a los servicios de urgencia para recibir un diagnóstico (Hurtado et al. 2020), y donde tan sólo un $25 \%$ de los pacientes son diagnosticados en entornos de AP (Piontek et al. 2018). 
Como ya se había identificado anteriormente (Bosman et al. 2016; Dickinson et al. 2010), nuestros resultados permitieron identificar que no todos los tratamientos son aceptados inicialmente, es más, existen barreras que provocan resistencia a comenzar con nuevos tratamientos, sobre todo en el caso de los fármacos, aunque esta resistencia inicial desaparece con el inicio del tratamiento, pudiendo reactivarse en momentos de crisis agudas. Sin embargo, en este estudio no han encontrado estas resistencias iniciales a los tratamientos psicológicos, como sí definen Berg, Sandahl y Bullington, 2010 y O’Brien et al., 2019. No obstante, una revisión sistemática cualitativa, en proceso de publicación, encontró que el proceso de aceptar una terapia psicológica dependía de la experiencia previa con la propia terapia, así como de las características del profesional sanitario y de las condiciones ambientales.

Los pacientes con ansiedad desean recibir información y asesoramiento (Prins et al. 2009) y nuestro análisis indica que la atención recibida desde AP no se valora positivamente, debido en parte, al poco tiempo empleado en consulta, a la falta de acompañamiento en momentos de crisis y a la falta de un plan de actuación establecido para el seguimiento de los tratamientos. Por último, las necesidades de información de las personas con TAG son en relación a los síntomas y los tratamientos disponibles. Los resultados de este estudio cualitativo han permitido integrar, junto a la síntesis de la evidencia cuantitativa de resultados de efectividad y seguridad de los tratamientos, y sobre la síntesis de la evidencia cualitativa, la perspectiva y experiencia de las personas con TAG en relación a los tratamientos, así como explicitar sus valores y preferencias, con el fin de acercar una medicina basada en la evidencia a las GPCs mediante un enfoque metodológico de estudios mixtos.

La síntesis de resultado final permitió aportar evidencia de suficiente calidad metodología, para integrar las recomendaciones finales de la GPC para el TAG en AP. Una limitación importante fue que no se cubrieron los perfiles para conseguir una máxima variabilidad, ya que sólo se realizó una entrevista a una persona de 65 años o más y sólo 2 personas con un diagnóstico reciente.

Tampoco se ha podido llegar a una saturación de la información. Los temas surgidos a partir de las entrevistas se podrían enriquecer a partir de la ampliación de este estudio.

Agradecimientos. Se agradece la participación que todos los pacientes tuvieron en las entrevistas. 


\section{REFERENCIAS}

Alonso, Jordi, and Jean Pierre Lépine. 2007. "Overview of Key Data from the European Study of the Epidemiology of Mental Disorders (ESEMeD)." The Journal of Clinical Psychiatry 68 Suppl 2: 3-9.

American Psychiatric Association. 2013. "Diagnostic and Stadistical Manual of Mental Disorders: DSM-V (5th Ed.). Fifth Edit." Washington, DC: American Psychiatric Association.

Bados, Arturo. 2017. "Trastorno de Ansiedad Generalizada." http://hdl.handle.net/2445/115724.

Begum, Mursheda, Grant Lewison, Eva Wölbert, Karen Berg Brigham, Meryl Darlington, Isabelle Durand-Zaleski, and Richard Sullivan. 2020. "Mental Health Disorders Research in Europe, 2001-2018." Evidence Based Mental Health 23 (1): 15-20. https://doi.org/10.1136/ebmental-2019-300130.

Berg, Adrienne Levy, Christer Sandahl, and Jennifer Bullington. 2010. "Patients' Perspective of Change Processes in Affect-Focused Body Psychotherapy for Generalised Anxiety Disorder." Body, Movement and Dance in Psychotherapy 5 (2): 151-69. https://doi.org/10.1080/17432979.2010.494853.

Boeft, Madelon den, Jos W. R. Twisk, Berend Terluin, Brenda W. J. H. Penninx, Harm W. J. van Marwijk, Mattijs E. Numans, Johannes C. van der Wouden, and Henriette E. van der Horst. 2016. "The Association between Medically Unexplained Physical Symptoms and Health Care Use over Two Years and the Influence of Depressive and Anxiety Disorders and Personality Traits: A Longitudinal Study." BMC Health Services Research 16 (1): 100. https://doi.org/10.1186/s12913-016-1332-7.

Bosman, Renske C, Klaas M Huijbregts, Peter Fm Verhaak, Henricus G Ruhé, Harm Wj van Marwijk, Anton Jlm van Balkom, and Neeltje M Batelaan. 2016. "Long-Term Antidepressant Use: A Qualitative Study on Perspectives of Patients and GPs in Primary Care." British Journal of General Practice 66 (651): e708-19. https://doi.org/10.3399/bjgp16X686641.

Dickinson, Rebecca, Peter Knapp, Allan O House, Vandana Dimri, Arnold Zermansky, Duncan Petty, John Holmes, and David K Raynor. 2010. "Long-Term Prescribing of Antidepressants in the Older Population: A Qualitative Study." British Journal of General Practice 60 (573): e144-55. https://doi.org/10.3399/bjgp10X483913.

Entwistle, Vikki A., and lan S. Watt. 2013. "Treating Patients as Persons: A Capabilities Approach to Support Delivery of Person-Centered Care." The American Journal of Bioethics 13 (8): $29-39$. https://doi.org/10.1080/15265161.2013.802060.

García-Campayo, Javier, Fernando Caballero, María Perez, and Vanessa López. 2012. "Prevalence and Clinical Features of Newly Diagnosed Generalized Anxiety Disorder Patients in Spanish Primary Care Settings: The GADAP Study." Actas Espanolas de Psiquiatria $40 \quad$ (3): 105-13. http://www.ncbi.nlm.nih.gov/pubmed/22723129.

Grupo de Trabajo de la Guía de Práctica Clínica para el Manejo de Pacientes con Trastornos de Ansiedad en Atención Primaria. 2008. "Guía de Práctica Clínica Para El Manejo de Pacientes Con Trastornos de Ansiedad En Atención Primaria." Madrid. Plan Nacional para el SNS del MSC. Unidad de Evaluación de Tecnologías Sanitarias. Agencia Laín Entralgo: Guías de Práctica Clínica en el SNS: UETS No 2006/10; 2008.

Hurtado, María M., Amelia Villena, Amanda Vega, Gisela Amor, Clara Gómez, and José Miguel Morales- Asencio. 2020. "I Have Anxiety, but I Have Values and Preferences' Experiences of Users with Generalized Anxiety Disorder: A Qualitative Study." International Journal of Mental Health Nursing, January, inm.12690. https://doi.org/10.1111/inm.12690.

Institute of Medicine (IOM). 2001. Crossing the Quality Chasm. Washington, D.C. National Academies Press. pp 360

Légaré, France, Rhéda Adekpedjou, Dawn Stacey, Stéphane Turcotte, Jennifer Kryworuchko, lan D Graham, Anne Lyddiatt, et al. 2018. "Interventions for Increasing the Use of Shared Decision Making by Healthcare Professionals." The Cochrane Database of Systematic Reviews 7: CD006732. https://doi.org/10.1002/14651858.CD006732.pub4.

Meuldijk, D., I.V.E. Carlier, I.M. van Vliet, T. van Veen, R. Wolterbeek, A.M. van Hemert, and F.G. Zitman. 2016. "The Clinical Effectiveness of Concise Cognitive Behavioral Therapy with or without Pharmacotherapy for Depressive and Anxiety Disorders; a Pragmatic Randomized Controlled Equivalence Trial in Clinical Practice." Contemporary Clinical Trials 47 (March): 131-38. https://doi.org/10.1016/j.cct.2015.12.021. 
O’Brien, Katie, Nora O'Keeffe, Hannah Cullen, Aoife Durcan, Ladislav Timulak, and James McElvaney. 2019. "Emotion-Focused Perspective on Generalized Anxiety Disorder: A Qualitative Analysis of Clients' inSession Presentations." Psychotherapy Research 29 (4): 524-40. https://doi.org/10.1080/10503307.2017.1373206.

Parmentier, H., J. García-Campayo, and R. Prieto. 2013. "Comprehensive Review of Generalized Anxiety Disorder in Primary Care in Europe." Current Medical Research and Opinion 29 (4): 355-67. https://doi.org/10.1185/03007995.2013.770731.

Piontek, Katharina, Meike C. Shedden-Mora, Maria Gladigau, Amina Kuby, and Bernd Löwe. 2018. "Diagnosis of Somatoform Disorders in Primary Care: Diagnostic Agreement, Predictors, and Comaprisons with Depression and Anxiety." BMC Psychiatry 18 (1): 361. https://doi.org/10.1186/s12888-018-1940-3.

Prins, Marijn A., Peter F.M. Verhaak, Klaas van der Meer, Brenda W.J.H. Penninx, and M. Bensing, Jozien. 2009. "Primary Care Patients with Anxiety and Depression: Need for Care from the Patient's Perspective." Journal of Affective Disorders 119 (1-3): 163-71. https://doi.org/10.1016/j.jad.2009.03.019.

Ritchie, J., Lewis, J., Nicholls, C.M. Ormston, R. 2014. Qualitative Research Practice: A Guide for Social Science Students \& Researchers. 2nd ed. London: SAGE.

Stacey, Dawn, France Légaré, Krystina Lewis, Michael J Barry, Carol L Bennett, Karen B Eden, Margaret Holmes-Rovner, et al. 2017. "Decision Aids for People Facing Health Treatment or Screening Decisions." Cochrane Database of Systematic Reviews, April. https://doi.org/10.1002/14651858.CD001431.pub5.

Stein, Dan J., Carmen C. W. Lim, Annelieke M. Roest, Peter de Jonge, Sergio Aguilar-Gaxiola, Ali Al-Hamzawi, Jordi Alonso, et al. 2017. "The Cross-National Epidemiology of Social Anxiety Disorder: Data from the World Mental Health Survey Initiative." BMC Medicine 15 (1): 143. https://doi.org/10.1186/s12916-0170889-2.

Stein, Murray B, and Jitender Sareen. 2015. "Generalized Anxiety Disorder." Edited by Caren G. Solomon. New England Journal of Medicine 373 (21): 2059-68. https://doi.org/10.1056/NEJMcp1502514.

Villagrasa, B, B Olaya, R Lopez-Anton, C de la Cámara, A Lobo, and J Santabárbara. 2019. "Prevalence of Anxiety Disorder among Older Adults in Spain: A Meta-Analysis." Journal of Affective Disorders 246: 40817. https://doi.org/10.1016/j.jad.2018.12.087.

Weijden, Trudy van der, Antoine Boivin, Jako Burgers, Holger J Schünemann, and Glyn Elwyn. 2012. "Clinical Practice Guidelines and Patient Decision Aids. An Inevitable Relationship." Journal of Clinical Epidemiology 65 (6): 584-89. https://doi.org/10.1016/j.jclinepi.2011.10.007.

World Health Organization. 2017. "Mental Health in the Workplace." WHO. World Health Organization. 2017. https://www.who.int/mental_health/es/. 